\title{
Sensitivity and specificity of traditional testing methods to detect sensory impairment in the skin lesions of leprosy compared to Semmes-Weinstein monofilaments
}

\author{
ANITHA RAMACHANDRAN ${ }^{\mathrm{a}}$, LULUA SAFAR ${ }^{\mathrm{b}}$, \\ SARITA SASIDHARANPILLAI ${ }^{\mathrm{c}}$, \\ KIDANGAZHIYATHMANA AJITHKUMAR ${ }^{\mathrm{d}}$, \\ BIJU GEORGE ${ }^{\mathrm{e}}$ \& KEER ANKULANGARA DEVI ${ }^{\mathrm{f}}$ \\ ${ }^{a}$ MBBS student, Department of Dermatology, Govt. Medical College, \\ Kozhikode, Kerala, India \\ ${ }^{\mathrm{b}}$ MBBS, Department of Dermatology, Govt. Medical College, Kozhikode, \\ Kerala, India \\ ${ }^{\mathrm{c}} \mathrm{MD}$, Associate Professor, Department of Dermatology, Govt. Medical \\ College, Kozhikode, Kerala, India \\ ${ }^{\mathrm{d}}$ MD, Additional Professor, Department of Dermatology, Govt. Medical \\ College, Thrissur, Kerala, India \\ ${ }^{\mathrm{e}} M D$, Associate Professor, Department of Social and Preventive Medicine, \\ Govt. Medical College, Kozhikode, Kerala, India \\ ${ }^{\mathrm{f}} \mathrm{MD}$, Professor and Head, Department of Dermatology, Govt. Medical \\ College, Kozhikode, Kerala, India
}

Accepted for publication 2 January 2020

\section{Summary}

Objective: To determine the sensitivity and specificity of traditional testing methods to detect sensory deficit in the skin lesions of leprosy in comparison to Semmes Weinstein (SW) nylon monofilaments.

Design: After ethical clearance and written informed consent from study subjects, a consecutive series of 50 patients aged $>12$ years with leprosy skin lesions, who attended our institution, were evaluated in this cross sectional study.

Results: 74 skin lesions of 50 patients were evaluated. Considering the monofilament test result as the gold standard, the sensitivity to detect sensory impairment was $95.9 \%$ for the ballpoint pen, $97.3 \%$ for a sterile needle and $100 \%$ for cotton wool, and cold and warm water.

Limitation: Paucity of cases in the lepromatous spectrum of disease and the probable absence of early lesions of disease in the study group were the study limitations.

Conclusions: The cotton wool and ballpoint pen methods are easy to perform in the field and have comparable sensitivity and specificity to the blue Semmes

Correspondence to: Sarita Sasidharanpillai, Government Medical College, Kozhikode Kozhikode, Kerala, India (e-mail: saritasclt@gmail.com)

The work has been done in the Dept. of Dermatology, Govt. Medical College, Kozhikode, Kerala, India. 
Weinstein nylon monofilament to detect sensory impairment in skin lesions, especially in tuberculoid cases.

Keywords: Semmes Weinstein nylon monofilament, sensory impairment, cotton wool, ballpoint pen, sterile needle, warm water, cold water

\section{Introduction}

Despite the elimination of leprosy as a problem of public health importance, new cases continue to be reported. ${ }^{1}$ Early diagnosis and treatment is the recommended strategy to break the chain of disease transmission. ${ }^{1}$ Leprosy is diagnosed by the cardinal signs proposed by WHO. ${ }^{2}$ Patients having one of the three signs (asymptomatic hypopigmented or erythematous skin lesion with sensory impairment; thickened peripheral nerve with sensory impairment over the area supplied by the nerve; acid fast bacilli positivity in skin smear studies) are diagnosed to have leprosy. Hence detection of defective sensory perception is significant in diagnosing leprosy.

Peripheral centres generally use the tip of a ballpoint pen (to test light touch), while many tertiary referral centres make use of cotton wool (for light touch), as well as the point of a sterile needle (for pain), and cold $\left(25^{\circ} \mathrm{C}\right)$ and warm water $\left(40{ }^{\circ} \mathrm{C}-45^{\circ} \mathrm{C}\right.$ as per various studies) in test tubes (for temperature), to detect sensory impairment. ${ }^{3,4}$ But these traditional methods are not standardised.

Semmes Weinstein nylon monofilaments (SW filaments) are considered as sensitive and objective tools to detect sensory impairment in leprosy. ${ }^{5}$ The standard SW filament set comprises of precisely calibrated six monofilaments that are of equal length. They are colour coded and calibrated to produce varying forces of pressure when applied on the surface of skin (Table 1). ${ }^{5}$

Villarroel, et al. in their study on cutaneous sensation of leprosy lesions, documented the monofilaments to have a sensitivity and a specificity of $81.7 \%$ and $96.1 \%$, respectively while thermal sensory testing to have sensitivity of $90.2 \%$ and $92.2 \%$, respectively for warm and cold water and $100 \%$ specificity for both. But they employed a thermal sensory analyser to perform the testing. The size and cost of thermal sensory analysers rule out their routine use. The authors concluded their study with the opinion that smaller and cheaper devices that can assess thermal perception need to be developed and at present monofilaments have adequate validity as a diagnostic tool in leprosy. ${ }^{3}$

In a study comparing the ballpoint pen with the monofilaments to detect sensory perception of hands and feet, the former was found to be less reliable. ${ }^{6}$ Sensory nerve function impairment affecting hands and feet in leprosy is denoted as Grade1 disability. Prompt detection of Grade1 disability and the adoption of protective life style practices may prevent progression to Grade2 disability in those affected. Hence, testing specified points (corresponding to the nerves supplying the area) in hands and feet with SW nylon monofilaments (the green filament exerting $0.05 \mathrm{gm}$ force for hands and the purple filament exerting $2 \mathrm{gm}$ force for feet) is recommended. 5

Saunderson and Groenen, in their study of 594 leprosy patients identified sensory loss in a typical skin patch as the most important sign of early leprosy. ${ }^{7}$ This observation points to the importance of accurate detection of sensory deficit in the skin patch of suspected leprosy cases. But SW filaments, considered as the objective tool to detect sensory deficit are not easily available; moreover a certain level of expertise is needed for sensory testing using them. 
Table 1. Pressure imparted by Semmes Weinstein nylon monofilaments on application on skin surface

\begin{tabular}{lc}
\hline Colour of filament & Pressure on surface of skin on application of filament \\
\hline Green & $0.05 \mathrm{gm}$ \\
Blue & $0.2 \mathrm{gm}$ \\
Purple & $2 \mathrm{gm}$ \\
Red & $4 \mathrm{gm}$ \\
Orange & $10 \mathrm{gm}$ \\
Light red & $300 \mathrm{gm}$ \\
\hline
\end{tabular}

Sensory testing with them is time consuming as well. Hence examining all skin lesions of leprosy for sensory impairment with SW filaments becomes less practical, especially in a field setting. There is a dearth of studies comparing the efficacy of traditional methods to detect sensory impairment in skin lesions of leprosy with that of SW filaments.

In this setting we compared the sensitivity and specificity of traditional methods to detect sensory impairment in skin lesions of leprosy with that of Semmes Weinstein monofilaments.

\section{Methodology}

\section{STUDY DESIGN: CROSS SECTIONAL STUDY}

Fifty patients aged more than 12 years and manifesting skin lesions of leprosy (diagnosed as per clinical evaluation, skin smear study and histology analysis), who attended the Dermatology department of a tertiary care centre from 1.6.2019 were included consecutively in the study (irrespective of duration of treatment), after clearance from the Institutional ethics committee and written informed consent from individual patient.

Patients not willing to participate in the study and patients with peripheral neuropathy due to other causes such as hereditary neuropathies, Type 2 diabetes, trauma and carpal tunnel syndrome, were excluded.

According to a previous study, the sensitivity and specificity of SW monofilaments is $81.7 \%$ and $96.1 \%$ in detecting sensory nerve function impairment. ${ }^{8}$ Taking an accuracy of $15 \%$, the sample size was determined as a minimum of 50.

In this cross sectional study, a pre-set proforma was used to collect data pertaining to patient characteristics, evolution of disease, and clinical features.

Site, morphology and number of skin lesions and enlarged nerves were noted for each individual case. Skin smear and histopathology findings were documented. Patients were grouped according to the Ridley-Jopling classification of leprosy as per clinical, histological and skin smear findings. The treatment required by the patients was recorded.

Skin lesions of leprosy were tested using cotton wool (for uniform assessment cotton was passed through aluminium rod of $2 \mathrm{~cm}$ length and $1 \mathrm{~cm}$ inner diameter, care was taken to touch and not to stroke the cotton across the skin), the $0.7 \mathrm{~mm}$ tip of a Reynolds Brite ballpoint pen (keeping the testing hand supported to make it more stable, skin touched gently with the tip of pen kept perpendicular to the surface of skin so as to produce just a slight movement of skin, taking care not to stroke or produce deep dent), a sterile needle prick (touched lightly on the skin without causing bleeding) with 23 gauge needle, and warm and cold water at $42{ }^{\circ} \mathrm{C}$ and $25^{\circ} \mathrm{C}$ (measured using digital thermometer) respectively in $10 \mathrm{~mm}$ Cresil glass test tubes. The Semmes Weinstein monofilament testing was performed with green and blue filaments, providing $0.05 \mathrm{gm}$ and $0.2 \mathrm{gm}$ force respectively. ${ }^{5}$ Each filament was applied perpendicularly 
Table 2. Age and gender distribution of study group

\begin{tabular}{cccccc}
\hline \multirow{2}{*}{ Gender } & \multicolumn{5}{c}{ Age distribution of study group } \\
\cline { 2 - 5 } & $12-20$ years & $21-40$ years & $41-60$ years & Above 60 years & Total \\
\hline Male & $7(20.6 \%)$ & $11(32.4 \%)$ & $10(29.4 \%)$ & $6(17.6 \%)$ & $34(100 \%)$ \\
Female & $3(18.8 \%)$ & $8(50 \%)$ & $5(31.3 \%)$ & $0(0 \%)$ & $16(100 \%)$ \\
Total & $10(20 \%)$ & $19(38 \%)$ & $15(30 \%)$ & $6(12 \%)$ & $50(100 \%)$ \\
\hline
\end{tabular}

to the specified site with mild pressure until it formed a ' $C$ ' curve. The approximate time of contact with the skin during each application was kept as one second.

Sensory testing was carried out starting from the normal skin on the opposite side to the leprosy lesion, followed by nearby normal skin and then on skin lesion itself. If the stimulus was felt as less than that in normal skin or if the patient pointed to a site $2 \mathrm{~cm}$ away as the site of application of particular stimulus, it was considered as hypoaesthesia. Anaesthesia was defined as complete absence of perception of the stimulus. Inability or reduced ability to appreciate the stimulus applied by the blue monofilament was taken as evidence of anaesthesia and hypoaesthesia respectively, to the SW filament testing. The same examiner evaluated all the patients and a single set of monofilaments was used throughout the study.

In patients with multiple skin lesions, (affecting different body parts and of different morphologies), representative lesions for each body area (face, trunk, upper limb and lower limb) and for each particular morphology were tested for sensory impairment and the results were documented.

Throughout the sensory testing, the patient's view of the skin being tested was blocked. Motor nerve function impairment was diagnosed when the power recorded by voluntary muscle testing was $<5$ on the Medical Research Council scale. ${ }^{5,9}$

The data were entered into Microsoft Excel and analysed with SPSS Version 18. Sensitivity and specificity of sensory impairment detected by traditional methods were compared with that of blue SW monofilament (which was adopted as the gold standard).

\section{Results}

The study population comprised of 50 patients with skin lesions diagnostic of Hansen's disease (Table 2). Ages ranged from 10 years to 75 years (mean 38.4, standard deviation 16.3). Males outnumbered females with a male:female ratio of (2.1:1).

Duration of disease in study participants ranged from 2 weeks to five years (mean 1.57 years \pm 1.23 years).

Among the study group there were seven (14\%) cases of Tuberculoid (TT), 38 (76\%) cases of Borderline Tuberculoid (BT), two cases (4\%) each of Borderline Lepromatous (BL) and Lepromatous (LL) and one of (2\%) Indeterminate leprosy. Skin lesions were distributed on the face, trunk, upper limbs and lower limbs. The different types of lesions observed were hypopigmented or asteatotic patches, plaques and infiltrated plaques (Table 3). None of the study participants showed nodular lesions or vague, shiny hypopigmented macules of leprosy. Ear lobe infiltration was noted in two LL cases.

Seven patients $(14 \%)$ required PB treatment and the others $(86 \%)$ needed MB treatment.

Seventy four skin lesions in 50 study participants were evaluated for sensory perception. All patients showed normal perception of stimuli on normal skin, on the opposite side of the body, and on nearby normal skin, when tested with cotton wool, tip of a ballpoint pen, sterile needle, 
Table 3. Distribution and morphology of leprosy skin lesions

\begin{tabular}{lcccc}
\hline Sites affected & \multicolumn{3}{c}{ Morphological type } \\
\cline { 2 - 5 } & $\begin{array}{c}\text { Hypopigmented and/ or } \\
\text { asteatotic patch }\end{array}$ & Plaque & Infiltrated plaque & Total \\
\hline Face & $6(66.7 \%)$ & $2(22.2 \%)$ & $1(11.1 \%)$ & $9(100 \%)$ \\
Trunk & $7(58.3 \%)$ & $3(25 \%)$ & $2(16.7 \%)$ & $12(100 \%)$ \\
Upper limb & $24(85.7 \%)$ & $3(10.7 \%)$ & $1(3.6 \%)$ & $28(100 \%)$ \\
Lower limb & $19(76 \%)$ & $6(24 \%)$ & $0(0 \%)$ & $25(100 \%)$ \\
Total & $56(75.7 \%)$ & $14(18.9 \%)$ & $4(5.4 \%)$ & $74(100 \%)$ \\
\hline
\end{tabular}

water at different temperatures and the blue monofilament (0.2 gm). Only 20/50 (40\%) patients were able to perceive the green filament $(0.05 \mathrm{gm})$ on non-lesional skin. In all 20 patients (all BT) who were able to perceive green filament in non lesional skin, anaesthesia was recorded in lesions. None of the patients who failed to feel the green filament in non-lesional skin was able to feel it in lesional skin.

69/74 leprosy lesions tested (93.2\%) showed hypoaesthesia or anaesthesia to all modalities of testing (cotton wool, ballpoint pen, sterile needle, water at different temperatures and the blue monofilament) (Table 4).

Two cases who showed normal perception to the ballpoint pen stimulus, the lesions were located on upper limbs. One was, 31 year old female with BT and the lesion was found to be anaesthetic to all other stimuli. The second patient was sixty year old female of BL spectrum, whose upper limb lesion showed hypoaesthesia for all methods except testing by ballpoint pen.

The face lesion of one 22 year old female patient with BT showed normal sensory perception to both ballpoint pen and sterile needle stimuli; but hypoaesthesia was noted to testing by temperature, cotton and blue monofilament (Table 4).

Among the 74 lesions tested with blue filament, 62 (83.8\%) were anaesthetic, $11(14.9 \%)$ hypoaesthetic and one (1.4\%) had normal sensory perception (Table 5).

On analysing the cutaneous sensation (normal/ hypoaesthesia/ anaesthesia) as assessed by different methods, we observed cotton wool (87.8\%) and water at different temperatures $(83.8 \%)$ to have greater concordance (than sterile needle and ballpoint pen) with the same recorded by blue filament (Table 6).

But all traditional methods were found to have good sensitivity to differentiate sensory impairment from normal sensory perception (Table 7).

The sensitivity to detect sensory impairment was $95.9 \%$ for tip of ballpoint pen, $97.3 \%$ for sterile needle and $100 \%$ for cotton wool and cold and warm water when compared to blue monofilament (Table 7). No significant variation was documented in sensitivity of different testing methods to detect sensory impairment with respect to morphology or site of the lesions.

Since all testing methods except cotton wool identified the normal sensory perception in the lone lesion that showed normal perception of blue filament, specificity to detect sensory impairment was $100 \%$ for all traditional methods except cotton wool.

\section{Discussion}

We observed all traditional methods to have high sensitivity (95.9\% for tip of ballpoint pen, $97.3 \%$ for sterile needle and $100 \%$ for cotton wool and cold and warm water) to detect sensory impairment in lesional skin of leprosy in comparison to blue SW filament. Moreover all stimuli had $100 \%$ specificity in making this distinction except cotton wool, which failed to identify 


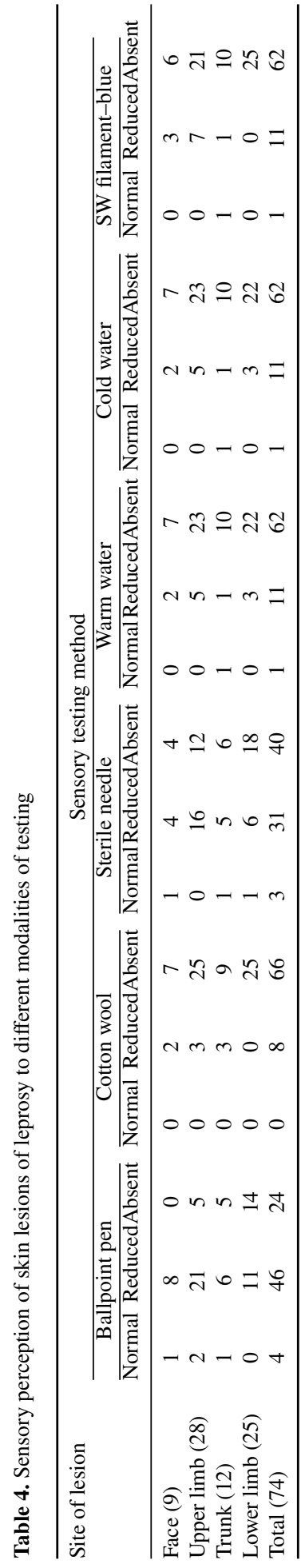




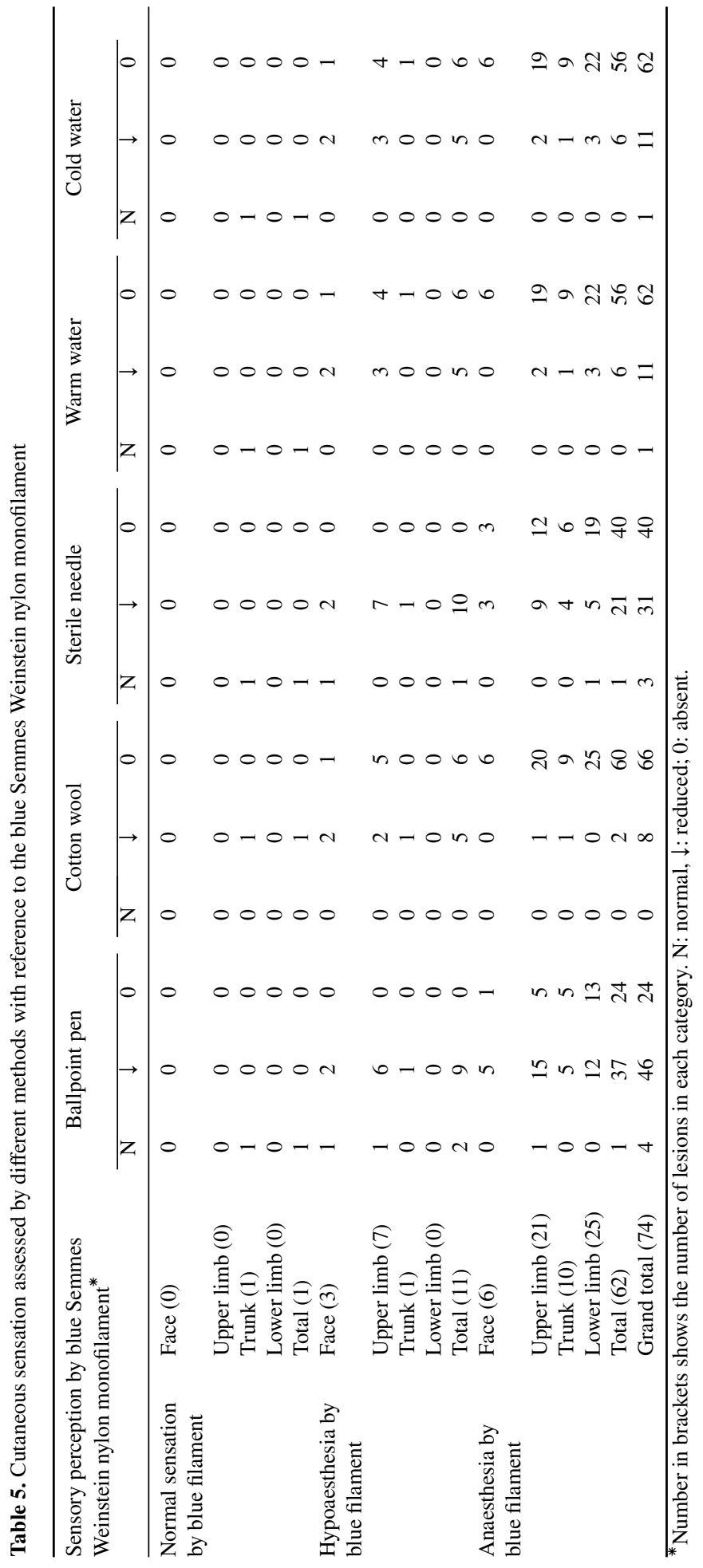


Table 6. Concordance between cutaneous sensation documented by individual traditional method and blue Semmes Weinstein nylon monofilament.

\begin{tabular}{lccccc}
\hline $\begin{array}{l}\text { Cutaneous sensation } \\
\text { as assessed by blue } \\
\text { filament }\end{array}$ & $\begin{array}{c}\text { Concordance } \\
\text { rate for } \\
\text { ballpoint pen }\end{array}$ & $\begin{array}{c}\text { Concordance } \\
\text { rate for cotton } \\
\text { wool }\end{array}$ & $\begin{array}{c}\text { Concordance } \\
\text { rate for sterile } \\
\text { needle }\end{array}$ & $\begin{array}{c}\text { Concordance } \\
\text { rate for warm } \\
\text { water }\end{array}$ & $\begin{array}{c}\text { Concordance } \\
\text { rate for cold } \\
\text { water }\end{array}$ \\
\hline Normal sensation (1) & $1 / 1$ & $0 / 1$ & $1 / 1$ & $1 / 1$ & $1 / 1$ \\
Hypoaesthesia (11) & $(100 \%)$ & $(0 \%)$ & $(100 \%)$ & $(100 \%)$ & $(100 \%)$ \\
& $9 / 11$ & $5 / 11$ & $10 / 11$ & $5 / 11$ & $5 / 11$ \\
Anaesthesia (62) & $(81.8 \%)$ & $(45.5 \%)$ & $(90.9 \%)$ & $(45.5 \%)$ & $(45.5 \%)$ \\
Total (74) & $24 / 62$ & $60 / 62$ & $40 / 62$ & $56 / 62$ & $56 / 62$ \\
& $(38.7 \%)$ & $(96.8 \%)$ & $(64.5 \%)$ & $(90.3 \%)$ & $(90.3 \%)$ \\
& $34 / 74$ & $65 / 74$ & $51 / 74$ & $62 / 74$ & $62 / 74$ \\
\end{tabular}

Table 7. Sensitivity of traditional method to detect sensory impairment in skin lesions of leprosy in comparison to Semmes Weinstein nylon blue monofilament

\begin{tabular}{lccccc}
\hline * Site of lesion & \multicolumn{5}{c}{ Sensory testing method } \\
\cline { 2 - 5 } & Ballpoint pen & Cotton wool & Sterile needle & Warm water & Cold water \\
\hline Face (9) & 8 & 9 & 8 & 9 & 9 \\
Upper limb (28) & $88.9 \%$ & $100 \%$ & $88.9 \%$ & $100 \%$ & $100 \%$ \\
& 26 & 28 & 28 & 28 & 28 \\
Trunk (11) & $92.9 \%$ & $100 \%$ & $100 \%$ & $100 \%$ & $100 \%$ \\
& 11 & 11 & 11 & 11 & 11 \\
Lower limb (25) & $100 \%$ & $100 \%$ & $100 \%$ & $100 \%$ & $100 \%$ \\
& 25 & 25 & 24 & 25 & 25 \\
Total (73) & $100 \%$ & $100 \%$ & $96 \%$ & $100 \%$ & $100 \%$ \\
& 70 & 73 & 71 & 73 & 73 \\
\hline
\end{tabular}

* Number in brackets shows the total number of lesions in each category detected to have sensory impairment by blue monofilament. The lone lesion that showed normal sensory perception to blue Semmes Weinstein filament is excluded from this table.

the normal sensory perception in the single lesion that manifested neither anaesthesia nor hypoaesthesia to testing by blue filament.

Cotton wool $(87.8 \%)$ and water at different temperatures $(83.8 \%)$ showed greater concordance with blue filament with respect to the grades of sensory impairment (Table 6) in comparison to ballpoint pen (45.9\%) and sterile needle $(68.9 \%)$. But this may not have much practical implication since when hypoesthesia and anaesthesia were considered together all these methods have high sensitivity in making a diagnosis of leprosy (Table 7).

Most of the studies involving SW filament have assessed the sensory perception pertaining to hands and feet since sensory nerve function impairment affecting these sites amounts to Grade1 disability; hence testing these sites requires a more sensitive test for early detection. ${ }^{6,8}$ But previous authors have demonstrated the importance of accurate detection of sensory impairment in the skin lesions for early diagnosis of leprosy. ${ }^{7}$ The literature suggests that the threshold for normal sensation for any part of the body is $0.05 \mathrm{gm}$ (green filament) except for the sole of foot where it is considered as $2 \mathrm{gm} .{ }^{5}$ Hence we adopted the inability to perceive 
the stimulus applied with blue monofilament $(0.2 \mathrm{gm})$ as the cut off for sensory nerve function impairment in the skin lesions of leprosy.

Adopting the blue filament rather than the green filament as the gold standard is supported by our observation of $30 / 50$ patients $(60 \%)$ failing to perceive green filament in unaffected skin. The false positivity to testing was minimised by comparing the ability to perceive sensation within lesions, with that of the corresponding area on the contralateral side, as well as on adjacent normal skin, and to document hypoaesthesia and anaesthesia when the sensory perception on lesions was less or absent respectively when compared to normal skin.

The lower sensitivity documented for ballpoint pen and sterile needle prick to detect sensory impairment of lesions on the face compared to other areas is explained by the fact that thin skin and overlapping and rich nerve supply of facial skin may mask the sensory deficit. Moreover, the tendency of the clinician to apply the tip of ballpoint pen and sterile needle more gently on face so as not to traumatise the skin might have also contributed.

The significance of the present study lies in the importance assigned to detection of sensory impairment in diagnosing leprosy. ${ }^{7}$ The most important and time tested strategy of prevention of transmission of leprosy is by early diagnosis and treatment of affected cases.

Traditional testing methods that assess touch, pain and temperature in suspected leprosy cases (by tip of ballpoint pain, cotton wool, pin prick and warm and cold water in test tubes, respectively) in field camps and hospitals are not standardised. When warm and cold water containing test tubes are used, the temperature imparted to skin at the point of application varies with the type of test tube used and also with the time interval between collection of water in test tubes and its application to skin. Sensory perception with the ballpoint pen, cotton wool and pin prick depend on the force with which the stimulus is applied.

Despite temperature being the early affected sensation in leprosy, Semmes Weinstein nylon monofilaments are considered as an objective and sensitive tool to detect sensory impairment, since instruments like thermal sensory analysers that can ensure proper testing of thermal sensations are not routinely available.

The paucity of BL and LL cases (who usually manifest minimal sensory impairment or normal sensation) and most of the cases belonging to tuberculoid group, may have resulted in the high rate of sensory impairment in the study. Hence the ability of different methods to distinguish minimal sensory deficit from normal perception could not be well assessed. Saunderson and Groenen, in their study noted lack of sensory impairment in skin lesions in $49 \%(132 / 248)$ of smear positive leprosy cases. ${ }^{7}$

The specificity of standardised cotton wool to differentiate sensory impairment from normal perception needs to be further evaluated in large studies including more cases belonging to different groups of the leprosy spectrum, since the former over diagnosed sensory impairment in the lone lesion that showed normal sensation with the blue filament.

The study indicates that the combination of standardised cotton wool and ballpoint pen could increase the sensitivity and specificity as well as maintaining an acceptable level of concordance with blue SW filament to detect the presence and extent of sensory impairment in skin lesions of leprosy.

Though water at different temperatures yielded comparable results to that of cotton wool in detecting sensory deficit and the extent of sensory impairment in lesional skin of leprosy, we recommend the use of standardised cotton wool over warm and cold water since the use of water at different temperatures for sensory testing may have practical difficulties in a field setting. Though sterile needle and ballpoint pen had equal specificity to detect sensory impairment, 
ballpoint pen may be the preferred option over sterile needle considering the latter's potential to cause injury and the need for replacement after each use.

We suggest testing with cotton wool (100\% sensitivity) which is confirmed by ballpoint pen ( $100 \%$ specificity) will be enough in routine clinical examination of skin lesions of leprosy and we can do away with heat and cold, needle and SW filament in the diagnosis of leprosy.

\section{Limitation}

As we had only a few cases from the Lepromatous and Indeterminate groups, the applicability of our results in these groups must be evaluated with larger studies.

\section{Conclusion}

Ballpoint pen and cotton wool stimuli are easy to perform methods in the field scenario that have comparable sensitivity and specificity to blue Semmes Weinstein nylon monofilament to detect sensory impairment in skin lesions of leprosy, especially in tuberculoid patients.

\section{DECLARATION}

The authors declare that this article has not been published in its present form in any other scientific journal.

\section{Acknowledgements}

The first author expresses sincere gratitude to the research committee of the institution for encouraging research among undergraduate medical students.

\section{Copyright}

The authors agree that the article is offered to Leprosy Review alone, that it will be subject to editorial revision and that its copyright becomes the property of Leprosy Review alone.

\section{Competing interest statement}

All authors declare that the answers to the questions on your competing interest form are all no and therefore have nothing to declare.

\section{Details of ethics approval}

Ethics committee clearance was obtained from the institutional ethics committee of Government Medical College, Kozhikode, Kerala, India on 19.2.2019.

\section{References}

1 WHO. Global leprosy update, 2016: accelerating reduction of disease burden. Weekly Epidemiological Record No. 35. 2017; 92: 501-520.

2 Training manual for medical officers: NLEP. Chapter 7. Classification and management of leprosy. Directorate of Health Services, Ministry of Health and Family Welfare, New Delhi. Available from: htp://nlep.nic.in/training.

3 Villarroel MF, Orsini MBP, Lima RC, Antunes CMF. Comparative study of the cutaneous sensation of leprosysuspected lesions using Semmes-Weinstein monofilaments and quantitative thermal testing. Lepr Rev, 2007; 78: 102-109.

4 Sasidharanpillai S, Binitha MP, Riyaz N, Ambooken B, Reena Mariyath OK, George B et al. Childhood leprosy: A retrospective descriptive study from Government Medical College, Kozhikode, Kerala, India. Lepr Rev, 2014; 85: 100-110. 
5 Rao PN, Suneetha SK, Ebenezer GJ. Neuritis: Definition, Clinicopathological manifestations and proforma to record nerve impairment in leprosy. In: Kumar B, Kar HK (eds), IAL Textbook of Leprosy. 2nd, New Delhi: Jaypee Publisher 2016.

6 Koelewijn LF, Meima A, Broekhuis SM, Richardus JH, Mitchell PD, Benbow C et al. Sensory testing in leprosy: comparison of ballpoint pen and monofilaments. Lepr Rev, 2003; 74: 42-52.

7 Saunderson P, Groenen G. Which physical signs help most in the diagnosis of leprosy? A proposal based on experience in the AMFES project, ALERT, Ethiopia. Lepr Rev, 2000; 71: 34-42.

8 Owen BM, Stratford CJ. Assessment of the methods available for testing sensation in leprosy patients in a rural setting. Lepr Rev, 995; 66: 55-62.

9 Brandsma JW, van Brakel WH. Protocol for motor function assessment in leprosy and related research questions. Ind J Lepr, 2001; 73: 145-158. 